\title{
Beamformed Flow-Acoustic Correlations in High-Speed Jets
}

\author{
Dimitri Papamoschou ${ }^{1}$ \\ University of California, Irvine, California 92691 \\ Philip J. Morris ${ }^{2}$ and Dennis K. McLaughlin ${ }^{3}$ \\ Penn State University, University Park, Pennsylvania 16802
}

An experimental study of simultaneous multi-point measurements in the flow and acoustic fields of a Mach 1.75 cold air jet is presented. A series of four optical deflectometer (OD) probes were used for the flow field measurements, and eight microphones arranged on a circular arc recorded the far-field pressure in the direction of peak emission. In addition, some of the OD probes were positioned in the near acoustic field of the jet. The correlation methodology involves calculating the delay-and-sum beamformer outputs of the OD probes and microphones, then computing the coherence between the two outputs. With the OD probes in the jet shear layer, there is a significant correlation, on the order of 0.1 , between the turbulent fluctuations and far-field noise in the direction of peak emission. As the OD probe moves transversely away from the jet, the coherence with the far-acoustic field first drops and then increases significantly to levels on the order of 0.3 . The drop is associated with the probe moving into the hydrodynamic pressure field that does not radiate to the far field; the subsequent increase signifies the probe being located in the acoustic pressure field. In the vicinity of the jet exit, the peak coherence between the OD signal and microphone beamformer coincides with the physical location of the OD probe. However, as the shear layer thickens downstream, the peak coherence generally lags the probe location. It is argued that this is caused by the refraction of the acoustic rays by the mean velocity and speed-of-sound gradients. The effect is particularly severe when the OD probe is on the jet centerline past the end of the potential core. This underscores the need to include refraction effects in calculating the beamformer output of the microphone array.

\section{Introduction}

$\mathrm{T}$ The experimental results presented in this paper represent part of a general effort to understand and model the turbulent noise sources in high-speed jets. Of primary interest is noise in the peak radiation direction which arises from the supersonic motion of instability waves or large-scale turbulent structures ${ }^{1-6}$. The approach involves flow-acoustic correlations using simultaneous, multi-point measurements of turbulent fluctuations in the jet and pressure fluctuations in the acoustic far field. This approach can be considered as an extension of the seminal works by Panda et al. ${ }^{7,8}$ in which density fluctuations at a single point in the flow were correlated with the signal of a microphone placed in the acoustic far field. Their work showed the high degree of correlation between the large scale turbulent structures and the far field noise radiated in the downstream quadrant of supersonic jets.

A natural extension of the past flow-acoustic correlation works is to use multiple, simultaneously-sampled probes in both the flow and acoustic fields. Multiple probes (microphones) in the acoustic field allow noise source location using beamforming methods. The use of multiple microphones for noise source location goes back to the polar correlation method by Fisher et al. ${ }^{9}$ and the study of phased-array beamforming by Billingsley and Kinns ${ }^{10}$. Since then, phased arrays have found growing application in the detection of airframe noise ${ }^{11,12}$ and jet noise ${ }^{13-16}$. Identification of the jet noise source is particularly challenging because it requires modeling of the noise source distribution ${ }^{9}$. One realizes that the only way to accurately detect the jet noise source from far-field measurements is to already know the source! Any number of models may fit the far-field observations. To make tangible progress, the far-field measurements must be accompanied by turbulence measurements in the flow. These measurements provide additional information that may aid in the construction of physically meaningful models for the noise source. Relevant quantities are the eddy correlation length scales and the eddy convective velocities. Both require

\footnotetext{
${ }^{1}$ Professor, Department of Mechanical and Aerospace Engineering, 4200 Engineering Gateway; dpapamos@uci.edu. Fellow AIAA.

${ }^{2}$ Boeing /A. D. Welliver Professor, Department of Aerospace Engineering, 233C Hammond Building; pjm@psu.edu. Fellow AIAA.

${ }^{3}$ Professor, Department of Aerospace Engineering, 230A Hammond Building; dkm2@psu.edu. Fellow AIAA.
} 
the simultaneous measurement of turbulent fluctuations using at least two probes. Such measurements are very challenging in high-speed jets. The hot-wire measurements in supersonic jets by Troutt and McLaughlin ${ }^{2}$ produced important time resolved data in unheated jets operating at reduced Reynolds numbers in a low pressure environment.

Advances in high speed flow-field instrumentation developed in the last ten years have opened new opportunities for experimentalists. The recent Particle Image Velocimetry (PIV) measurements in compressible heated jets by Bridges ${ }^{16}$ and his co-workers have been able to obtain space-time velocity correlation data for a limited number of high speed jets. Within the same NASA organization, Panda and Seasholtz ${ }^{8}$ have developed a unique Rayleigh scattering instrumentation system for the measurement of density and velocity fluctuations. In their most recent work, Panda et al. ${ }^{7}$ have used their Rayleigh scattering instrument to measure correlations between velocity fluctuations in the jet and signals of microphones placed in the acoustic far field. These data have shown the high degree of correlation between the large scale turbulent structures and the far field noise radiated in the downstream quadrant of supersonic jets. Hileman et al. ${ }^{17}$ used a combination of microphone beamforming (done mostly in time domain) and flow visualization to study the connection between highly energetic turbulence and noise events in high-speed jets; our study shares some similarities with their approach. Finally, application of the optical deflectometer to high speed jets by Doty and McLaughlin ${ }^{18,19}$ and Petitjean et al. ${ }^{20}$ has produced extensive data on two point space-time correlations that cover a substantial spatial extent of jets over a wide range of Mach numbers. Although this instrument measures correlations in density gradients in the flow, it has been shown ${ }^{19}$ that such data measured in high speed jets are equivalent to velocity fluctuation correlation data measured by a number of prior investigators in low speed jet flows. It is this technique that is applied here for multiple-probe measurements in the flow.

This paper provides an initial framework for combining simultaneous, multi-point measurements in the flow and acoustic far field into a method for the characterization the jet noise sources. The approach is based on beamforming both the microphones and the flow probe signals, then cross-correlating the two beamformed outputs. The overall approach is illustrated in Fig. 1. Initial results for an unheated Mach 1.75 jet are reported.

\section{Experimental Setup}

\section{A. Flow Conditions}

The experiments at U.C. Irvine were performed on perfectly expanded round jets of Mach numbers 0.9 , 1.5, and 1.75. The supersonic jets were unheated while the subsonic jet was operated at a simulated heated condition (using helium-air mixtures) so that its velocity matched the velocity of the $M=1.5$ unheated jet. Here, results for the $M$ $=1.75$ jet with velocity $U=470 \mathrm{~m} / \mathrm{s}$ and estimated convective Mach number $M_{\mathrm{c}}=1.2$ are reported. The jet was produced by a 12.7-mm nozzle designed by the method of characteristics. The nozzle was connected to the jet apparatus shown in Fig. 2a and was supplied by unheated air at a nozzle pressure ratio 5.3, resulting in a perfectly expanded flow. The jet Reynolds number was 670,000.

\section{B. Diagnostics}

Noise measurements were performed in the aeroacoustic facility shown in Fig. 2b. The microphone array consisted of eight 3.2-mm condenser microphones (Bruel \& Kjaer, Model 4138) arranged on a circular arc centered in the vicinity of the nozzle exit. The polar aperture of the array was $30^{\circ}$ and the array radius was $1 \mathrm{~m}$. The angular spacing of the microphones was logarithmic. The entire array structure was rotated around its center to place the array at the desired polar angle. The arrangement of the microphones inside the anechoic chamber, and the principal electronic components, are shown in Fig. 2b. The microphones were connected, in groups of four, to two amplifier/signal conditioners (Bruel \& Kjaer, Nexus 2690-A-OS4) with a high-pass filter set at $300 \mathrm{~Hz}$ and a low-pass filter set at $100 \mathrm{kHz}$.

The basic operation of the optical deflectometer is illustrated in Fig. 3. The present setup used four probes, two stationary and two moveable (Fig.1), with four avalanche photo diodes (APDs). This is a new system that was designed, fabricated and tested at Penn State before being shipped to U.C. Irvine for operation. A detailed description of the system can be found in a companion paper by Veltin et $\mathrm{al}^{22}$.

The eight microphone signals and four optical-deflectometer outputs (twelve channels total) were sampled simultaneously at $160 \mathrm{kHz}$ by two multi-function data acquisition boards (National Instruments PCI-6070E) 
installed in a Pentium 4 personal computer. The maximum resolved frequency was thus $80 \mathrm{kHz}$. National Instruments LabView software was used to acquire the signals.

\section{Correlation Methodology}

\section{A. Correlation between an individual OD probe and a beamformed microphone array (OD-DAS $\left.{ }_{\text {mic }}\right)$}

The correlation between the optical deflectometer (OD) signal with the output of the focused array is illustrated in Fig. 4. The purpose of these experiments is to investigate correlations between the deflectometer signal and the farfield pressure signal emanating from the vicinity of the probe volume of the deflectometer. To focus the array at a certain point $\vec{\xi}$, the common delay-and-sum (DAS) method is used:

$$
g(\vec{\xi}, t)=\sum_{m=1}^{M} p_{m}\left(t+\tau_{m}(\vec{\xi})\right)
$$

with $p_{m}(t)$ the signal of microphone $m$ and $\tau_{m}(\vec{\xi})$ the acoustic propagation time from microphone $m$ to point $\vec{\xi}$. The time delay is based on a straight path between focus point $\vec{\xi}$ and microphone $m$. The OD probe is located at point $\vec{x}$ and measures the signal $f(\vec{x}, t)$. The cross-spectrum of the OD and $\mathrm{DAS}_{\text {mic }}$ signals is given by,

$$
S_{f g}(\vec{x}, \vec{\xi}, \omega)=\left\langle F(\vec{x}, \omega) G^{*}(\vec{\xi}, \omega)\right\rangle
$$

where $F$ and $G$ are the Fourier transforms of $f$ and $g$, respectively. From Eq.1, the Fourier transform of the DAS ${ }_{\text {mic }}$ signal is

$$
G(\vec{\xi}, \omega)=\sum_{m=1}^{M} P_{m}(\omega) e^{i \omega \tau_{m}(\vec{\xi})}
$$

where $P_{m}$ is the Fourier transform of $p_{m}$. Substitution into Eq.2 gives,

$$
S_{f g}(\vec{x}, \vec{\xi}, \omega)=\sum_{m=1}^{M}\left\langle F(\vec{x}, \omega) P_{m}^{*}(\omega)\right\rangle e^{-i \omega \tau_{m}(\vec{\xi})}
$$

To understand the correlations between flow and acoustics, it is important to present this cross-spectrum in a nondimensional form. To do this, the autospectrum of the OD signal is first computed from,

$$
S_{f f}(\vec{x}, \omega)=\left\langle F(\vec{x}, \omega) F^{*}(\vec{x}, \omega)\right\rangle
$$

and the autospectrum of the DAS signal, often referred to as the array beamformer output is given by,

$$
S_{g g}(\vec{\xi}, \omega)=\left\langle G_{n}(\vec{\xi}, \omega) G_{m}^{*}(\vec{\xi}, \omega)\right\rangle=\sum_{m=1}^{M} \sum_{n=1}^{M}\left\langle P_{n}(\omega) P_{m}^{*}(\omega)\right\rangle e^{i \omega\left[\tau_{n}(\vec{\xi})-\tau_{m}(\vec{\xi})\right]}
$$

Now the coherence of the OD-DAS ${ }_{\text {mic }}$ signals is defined as follows:

$$
\gamma_{f g}^{2}(\vec{x}, \vec{\xi}, \omega)=\frac{\left|S_{f g}(\vec{x}, \vec{\xi}, \omega)\right|^{2}}{S_{f f}(\vec{x}, \omega) S_{g g}(\vec{\xi}, \omega)}
$$

In this paper results on the OD-DAS ${ }_{\text {mic }}$ cross-spectra with the OD probe at various points inside and outside the $\mathrm{M}=1.75$ jet are presented.

\section{B. Correlation between beamformed OD signals and beamformed microphone array (DAS $\left.{ }_{O D}-D A S_{\text {mic }}\right)$}

To construct a single signal out of the four OD probes (when they are aligned in the axial direction), a "delay-andsum” method is used. This involves time delays based on the convective velocity $U_{c}$. The convective velocity can be determined using space-time correlations of the OD probe signals or, as will be shown below, by maximizing the value of the delay-and-sum variance. Consider the axial arrangement of Fig.5. The OD probes are at positions 
$x+\Delta x_{j}$, with $\Delta x_{1}=0$. Time and space are referenced to the position $x$ of the first probe. The delay-and-sum beamformer for the optical deflectometer $\left(\mathrm{DAS}_{\mathrm{od}}\right)$ is

$$
h(x, t)=\sum_{j=1}^{J} f_{j}\left(x, t+T_{j}\right), \quad T_{j}=\frac{\Delta x_{j}}{U_{c}}
$$

where $J$ is the total number of probes (in this case $J=4$ ). Before discussing the spectrum of $h(x, t)$, it is important to realize an important property of its variance

$$
\sigma_{h}^{2}(x)=\langle h(x, t) h(x, t)\rangle=\sum_{i=1}^{J} \sum_{j=1}^{J}\left\langle f_{i}\left(x, t+T_{i}\right) f_{j}\left(x, t+T_{j}\right)\right\rangle
$$

The summation comprises variances of the individual probe signals, which are constants, and cross-correlations between probe signals. The latter are in fact space-time correlations, maximized when $T_{j}=\Delta x_{j} / U_{c}$. Therefore, maximization of $\sigma_{h}^{2}$ offers an alternative means to determine the convective velocity $U_{c}$.

Consider the cross-spectrum of the DAS $S_{\text {mic }}$ and $\mathrm{DAS}_{\text {od }}$ signals, given respectively by Eqs.1 and 8. It is assumed that the OD probes and the beamformer focus of the microphone array fall on the same axial line (in this experiment the nozzle centerline or lip line), so the vector notation is omitted from the beamforming point. The DAS mic $-\mathrm{DAS}_{\text {od }}$ cross-spectrum is

$$
S_{h g}(x, \xi, \omega)=\left\langle H(x, \omega) G^{*}(\xi, \omega)\right\rangle
$$

From Eq.8, the Fourier transform of the DAS $_{\text {od }}$ signal is

$$
H(x, \omega)=\sum_{j=1}^{J} F_{j}(x, \omega) e^{i \omega T_{j}}
$$

Substitution of Eqs. 3 and 11 in Eq.10 yields,

$$
S_{h g}(x, \xi, \omega)=\sum_{j=1}^{J} \sum_{m=1}^{M}\left\langle F_{j}(x, \omega) P_{m}^{*}(\omega)\right\rangle e^{i \omega\left[\tau_{m}(\xi)-T_{j}\right]}
$$

Using steps similar to those for deriving the autospectrum of the DAS $S_{\text {mic }}$ signal, Eq.6, the autospectrum of the DAS $_{\text {od }}$ signal is

$$
S_{h h}(x, \omega)=\left\langle H \quad(x, \omega) H^{*}(x, \omega)\right\rangle=\sum_{j=1}^{J} \sum_{i=1}^{J}\left\langle F_{j}(x, \omega) F_{i}^{*}(\omega)\right\rangle e^{i \omega\left(T_{j}-T_{i}\right)}
$$

The coherence of the $\mathrm{DAS}_{\text {od }}-\mathrm{DAS}_{\text {mic }}$ signals is:

$$
\gamma_{h g}^{2}(x, \xi, \omega)=\frac{\left|S_{h g}(x, \xi, \omega)\right|^{2}}{S_{h h}(x, \omega) S_{g g}(\xi, \omega)}
$$

Using Parseval's theorem the variance can be evaluated using the autospectrum of Eq. 13, that is,

$$
\sigma_{h}^{2}(x)=\frac{1}{\pi} \int_{0}^{\infty} S_{h h}(x, \omega) d \omega
$$

Therefore the convective velocity can be determined by maximizing the integral of Eq.15.

\section{Results}

For all the results shown, the microphones were in located in the aft quadrant at polar angles ranging from 23.7 to 53.5 deg., as shown in Fig. 6. The optical deflectometer (OD) probes translated axially and normally in the jet and its vicinity. First, the microphone beamformer map for the $\mathrm{M}=1.75$ jet is shown. Figure 7 shows isocontours of the DAS $_{\text {mic }}$ autopectrum $S_{g g}$ (Eq. 6) on the $x / D-S r$ plane, where $S r=f D / U$ is the Strouhal number of the jet. The map 
shows a global peak at $x / D=12$ and $S r=0.15$. As the Strouhal number increases, the peak intensity moves toward the nozzle exit.

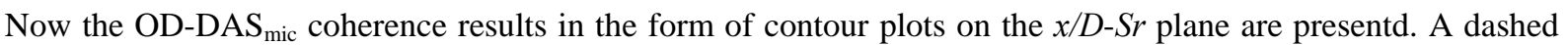
white line indicates the axial position of the reference OD probe. The same format will be used for all the coherence

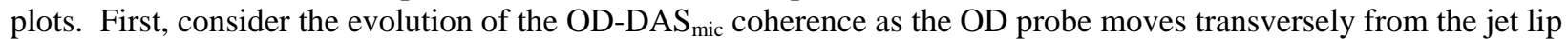
line to a position outside the jet flow. Figure 8 shows this evolution for the OD probe at $x / D=3.0$ for four radial positions of the OD. Also shown in the figure, below the transverse distance, is the probe location in terms of a similarity coordinate $(y-D / 2) / x$. This provides an indication of the relative location of the probes in the jet shear layer as the downstream distance changes. For reference, at $(y-D / 2) / x=0.1$, the axial turbulence intensity has fallen to $50 \%$ of its maximum value near $(y-D / 2)=0.0$ (see Davies $\left.{ }^{21}\right)$. With the OD probe close to the lip line $(y / D=0.48)$, a peak coherence of 0.06 is measured. This peak occurs about two jet diameters upstream of the probe location and at Strouhal number $S r=0.6$. One might suspect calibration errors for the mismatch between the coherence peak location and the probe locations, but, as discussed below, this is not the case. As the OD probe moves outside the jet flow, the coherence strengthens and its peak location moves toward the probe location. At $y / D=1.18$, the coherence peaks at 0.25 . Further movement of the probe outside the jet leads to a smaller coherence as the probe moves outside the region of peak noise emission. It is notable that, with the OD probe outside the jet, the peak coherence occurs exactly at the axial location of the OD probe. This indicates that the microphone array is properly aligned and calibrated, and that the spatial lag seen in Fig. 8 is connected to the jet flow field. As shown by Bogey and Bailly $^{23}$, the ray path connecting a point inside the jet to a point outside the jet is not a straight line; rather, it is bent inside the jet because of the gradients mean velocity and speed of sound. As a result, the time delay $\tau_{m}(\vec{\xi})$ used in the beamforming (Eq. 1) is different from that calculated using a straight ray path. This can cause shifting and blurring of focus from its intended position.

Similar phenomena are observed with the OD probe located at $x / D=5.5$ and 8.0, shown respectively in Figs. 9 and 10. One interesting feature that is more evident in these figures is the variation in the maximum coherence as the OD probe is moved outward from the lip line. Initially there is a decrease in the maximum coherence and then an increase to higher levels than obtained with the OD probe on the lip line. Though the reason for this behavior is not fully understood, an explanation can be found in the response of the OD. The OD senses fluctuations in the density gradient. These can be caused by velocity fluctuations that perturb the mean density gradient or by pressure fluctuations. On the jet lip line the velocity fluctuations are at their maximum and are probably the dominant cause of the fluctuations detected by the OD. In turn, these velocity fluctuations are dominated by the motions of the large scale turbulent structures which are known to correlate well with the noise radiation in the peak noise direction in high speed jets. Moving outwards from the jet lip line the velocity fluctuations decrease and initially the hydrodynamic pressure fluctuations will control the OD signal. Since the only a small fraction of the hydrodynamic pressure fluctuations radiate as sound the correlation with the far field pressure is likely to decrease. Further out from the jet lip line, well outside the edge of the jet, the OD senses the acoustic fluctuations, which are clearly well correlated with the far field sound. This explains the eventual increase in the coherence levels.

The clear advantage of the processing used in Figures 8 to 10 is seen by comparing the plots with similar OD to microphone correlations performed without the use of the beamformer with the microphone array. Figure 11 displays isocontours of the coherence of the single OD probe traversed along the lip line of the jet correlated with a single microphone located at the approximate center of the microphone array. The resulting fidelity is nowhere near the clarity seen in the corresponding isocontours of the coherence of the OD and the beamformed microphone array output (shown in the top part of Figure 8). The simpler processing used in Figure 11, taken from Veltin et al. ${ }^{20}$, does produce some additional insights, but not with the same clarity that is evident in the results of Figs. 8-10 of the present paper.

Moving now to the DAS ${ }_{\text {od }}-D^{-} S_{\text {mic }}$ correlations, Fig.12 shows an example of the determination of $U_{c}$ by maximizing the variance of the OD beamformer, as discussed in section III.B. Figures 13 to 16 show DAS od $^{-}$ DAS $_{\text {mic }}$ coherences for various OD probe locations and compare them to the OD-DAS ${ }_{\text {mic }}$ coherences at the same reference probe location. For each case, the $\mathrm{DAS}_{\text {od }}$ is based on the convective velocity determined by maximization of its variance, Eq. 9. In Fig. 13, the reference OD probe is located on the lip line near the nozzle exit at $x / D=2.0$. So close to the nozzle exit, the shear layer thickness is too small to cause significant bending of the rays emanating from the probe location. As a result, the peaks of the coherences coincide with the probe location. The coherence

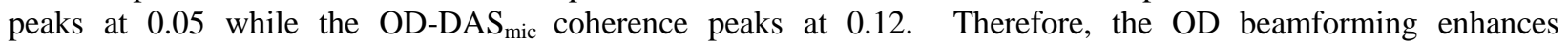
significantly the correlations with the microphone array. As the reference OD probe moves downstream along the lip line to $x / D=3.5$, Fig. 14, the shear layer thickens and refraction effects become more pronounced. As a result, the coherences peak about one jet diameter upstream of the reference OD probe location. The DAS ${ }_{\text {od }}-\mathrm{DAS}_{\text {mic }}$ 


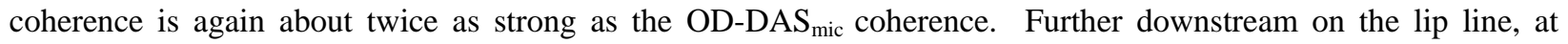
$x / D=10$ (Fig. 15), the refraction effects cause significant blurring of the coherence peak, but again the enhanced coherence of DAS $_{\text {od }}-\mathrm{DAS}_{\text {mic }}$ can be observed The doubling of the maximum coherence when the beamformed OD signal is used emphasizes the fact that the acoustic radiation in the peak noise direction is associated with turbulent structures that propagate at $U_{c}$ and, perhaps more importantly, are correlated over the relatively large axial separation of the OD sensors. This is strong evidence that the noise is dominated by coherent large scale turbulent structures.

When the reference probe is moved to the centerline of the jet at $x / D=10$, Fig.16, the coherence peaks move near the nozzle exit and the coherence pattern is severely smeared. This is believed to be due to the severe distortion of the ray paths emanating from a point on the centerline of the jet. This is a major effect that impacts the ability to accurately image noise sources. It underscores the need to account for refraction effects in noise source imaging methods. In addition, it should be noted that when the OD probe is interrogating the jet centerline the light beam will be influenced by fluctuations at all locations across the jet. This reflects the integration effect of the schlieren system.

Examination of the plots in Figs. 13-16 shows the reduction of the peak Strouhal number with axial distance, from $S r=0.6$ at $x / D=2.0$ to $S r=0.25$ at $x / D=10$. This shown in Fig. 17, where the Strouhal number peak obtained from Figs. 13-16 is shown as a function of downstream distance. These values are very consistent with "ridge" in the contours plot shown in Fig. 7 based on the beamformed microphone measurements alone.

\section{Conclusions}

Simultaneous multi-point measurements in the flow and acoustic fields of a Mach 1.75 cold air jet with supersonic convection velocity have been conducted. A series of four optical deflectometer OD probes were used for the flow field measurements, and eight microphones arranged on circular arc recorded the far-field pressure in the direction of peak emission. In addition, some of the OD probes were moved to the near acoustic field of the jet. The correlation methodology involves calculating the delay-and-sum beamformer outputs of the OD probes and microphones, then computing the coherence between the two outputs. The principal conclusions are as follows:

- With the OD probes in the jet shear layer, there is a significant correlation, on the order of 0.1 , between turbulence and far-field noise in the direction of peak emission.

- As the OD probe moves transversely away from the jet, the coherence with the far-acoustic field first drops and then increases significantly to levels on the order of 0.3. The drop is associated with the probe moving into the hydrodynamic pressure field that does not radiate to the far field; the subsequent increase signifies the probe being located in the acoustic pressure field.

- In the vicinity of the jet exit, the peak coherence between OD signal and microphone beamformer coincides with the physical location of the OD probe. However, as the shear layer thickens downstream, the peak coherence generally lags the probe location. It is believed that this is caused by the refraction of the acoustic rays by the mean velocity and speed-of-sound gradients. The effect is particularly severe when the OD probe is on the jet centerline beyond the end of the potential core. This underscores the need to include refraction effects in the calculation of the beamformer output of the microphone array.

- Correlation of the beamformed OD signal, using the measured convection velocity of the turbulence, with the microphone beamformed output produces isocontours with the greatest fidelity and with significantly higher coherence levels than obtained with single OD to microphone correlations. This method thus promises to produce data that will be more useful in assisting the development of predictive models for the various noise generation mechanisms.

- The increased coherence levels associated with the beamformed OD-microphone correlations indicates that the noise radiated in the peak noise radiation direction is associated with turbulence convecting at $U_{c}$, which is determined experimentally, and is coherent over a relatively large axial distance. This is additional strong evidence for the importance of the coherent large scale turbulent structures in noise radiation from high speed jets.

\section{Acknowledgments}

This work was performed under the sponsorship of the NASA NRA agreement NNX08AC44A monitored by Dr. James E. Bridges. The authors would like to thank Dr. Jeremy Veltin and Mr. Ben Day (Penn State) for designing 
the new optical deflectometer system and assisting in its implementation at U.C. Irvine; Mr. Salvador Mayoral (U.C. Irvine) for his design and construction of the support system for the deflectometer; and Messrs. Preben Nielsen, Andrew Johnson, and Vincent Phong (U.C. Irvine) for their assistance with the experiments.

\section{References}

1. McLaughlin, D.K., Morrison, G.L. and Troutt, T.R., "Experiments on the Instability Waves in a Supersonic Jet and Their Acoustic Radiation,” Journal of Fluid Mechanics, Vol. 69, 1975, pp. 73-85.

2. Troutt, T. R. and McLaughlin, D. K., "Experiments on the Flow and Acoustic Properties of a Moderate-Reynolds-Number Supersonic Jet,” Journal of Fluid Mechanics, Vol. 116, 1982, pp. 123-156.

3. Morris, P. J. "Flow Characteristics of the Large-Scale Wavelike Structure of a Supersonic Round Jet," Journal of Sound and Vibration, Vol. 53, No. 1, 1977, pp. 223-244.

4. Tam, C.K.W., "Directional Acoustic Radiation from a Supersonic Jet Generated by Shear Layer Instability,” Journal of Fluid Mechanics, Vol. 46, 1971, pp. 757-768.

5. Tam, C. K. W., “Supersonic Jet Noise Generated by Large Scale Disturbances, ” Journal of Sound and Vibration, Vol. 38, No. 1, 1975, pp. 51-79.

6. Tam, C. K. W. and Morris, P. J., “The Radiation of Sound by the Instability Waves of a Compressible Plane Turbulent Shear Layer,” Journal of Fluid Mechanics, Vol. 98, No. 2, 1980,pp. 349-381.

7. Panda, J., Seasholtz, R.G. and Elam, K.A., "Measurement of Correlation Between Flow Density and Velocity with Far Field Noise in High Speed Jets”, AIAA-2002-2485, Jan. 2002.

8. Panda, J., Seasholtz, R.G, "Experimental investigation of Density Fluctuations in High-Speed Jets and Correlation with Generated Noise”, Journal of Fluid Mechanics, Vol. 450, 2002, pp. 97-130.

9. Fisher, M.J., Harper-Bourne, M., and Glegg, S.A.L., “Jet Engine Source Location: The Polar Correlation Technique,” Journal of Sound and Vibration, Vol. 51, No.1, 1977, pp. 23-54.

10. Billingsley, J., and Kinns, R., “The Acoustic Telescope,” Journal of Sound and Vibration, Vol. 48, No. 4, 1976, pp. 485-510.

11. Humphreys, W.M., Brooks, T.F., Hunter, W.W., and Meadows, K.R., “Design and Use of Microphone Directional Arrays for Aeroacoustic Measurements,” AIAA-98-0471, 1998.

12. Brooks, T.F., and Humphreys, W.M., “A Deconvolution Approach for the Mapping of Acoustic Sources (DAMAS) Determined from Phased Microphone Arrays,” AIAA-2004-2954, 2004.

13. Lee, S.S., and Bridges, J., "Phased-Array Measurements of Single Flow Hot Jets," AIAA-2005-2842, 2005,

14. Venkatesh, S.R., Polak D.R., and Narayanan S., "Beamforming Algorithm for Distributed Source Localization and its Application to Jet Noise,” AIAA Journal, Vol. 41, No. 7, 2003, pp. 1238-1246.

15. Papamoschou, D., “Imaging of Distributed Directional Noise Sources,” AIAA-2008-2885, May 2008.

16. Bridges, J., “Effect of Heat on Space-Time Correlations in Jets”, AIAA-2006-2534, May 2006.

17. Hileman, J.I., Thurow, B.S., Caraballo, E.J., and Samimy, M., "Large-Scale Structure Evolution and Sound Emission in High-Speed Jets: Real-Time Visualization with Simultaneous Acoustic Measurements,” Journal of Fluid Mechanics, Vol. 544, 2005, pp. 277-307.

18. Doty, M. and McLaughlin, D.K., "Two-Point Correlations of Density Gradient Fluctuations in High Speed Jets Using Optical Deflectometry,” AIAA-2002-0367, Jan. 2002.

19. Doty, M.J., and McLaughlin, D.K., "Space-Time Correlation Measurements of High-Speed Axisymmetric Jets Using Optical Deflectometry,” Experiments in Fluids, Vol. 38, No. 25, pp. 415-425.

20. Petitjean, B., Viswanathan, K., McLaughlin, D.L., and Morris, P.J., 2007, "Space-Time Correlation Measurements in Subsonic and Supersonic Jets Using Optical Deflectometry,” AIAA-2007-3613, May 2007.

21. Veltin, J., Day, B., and McLaughlin, D.K., “Correlation of Flow and Acoustic Field Measurements in High Speed Jets,” AIAA-2009-3211, May 2009.

22. Davies, P. O. A. L., “Turbulence Structure in Free Shear Layers,” AIAA Journal, Vol. 4, No. 11, 1966, pp. $1971-1978$.

23. Bogey, C., and Bailly, C., "An Analysis of the Correlations between the Turbulent Flow and the Sound Pressure Fields of Subsonic Jets,” Journal of Fluid Mechanics, Vol. 583, 2007. pp. 71-97. 


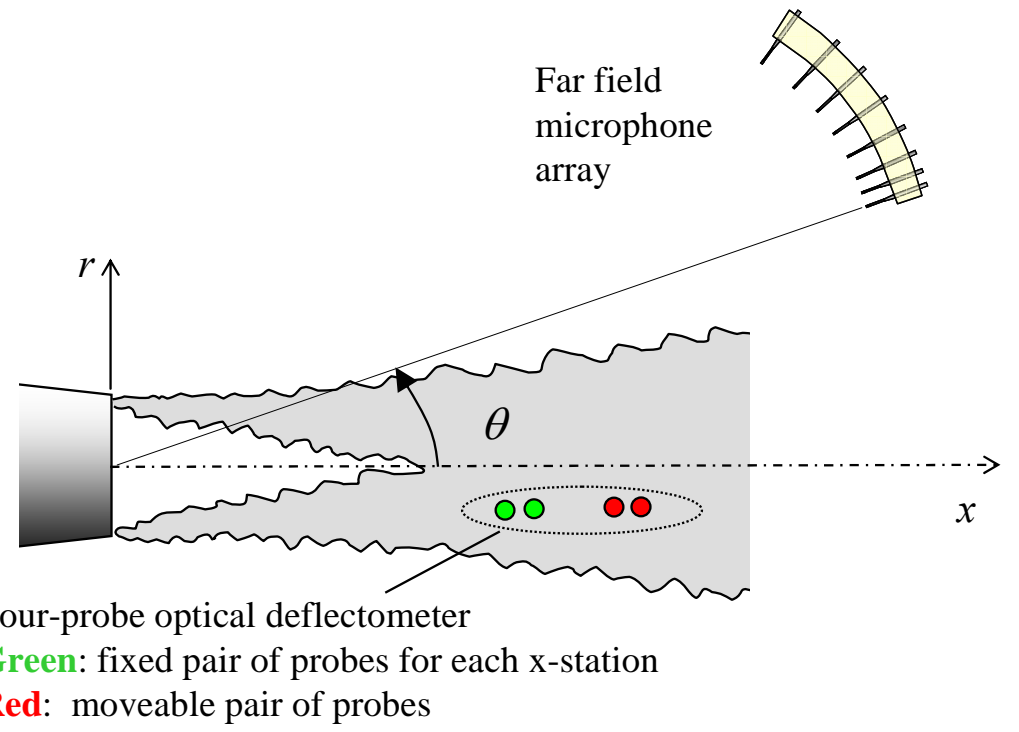

Fig. 1 Schematic of optical deflectometer (OD) and microphone array setup at the UCI lab.

a)

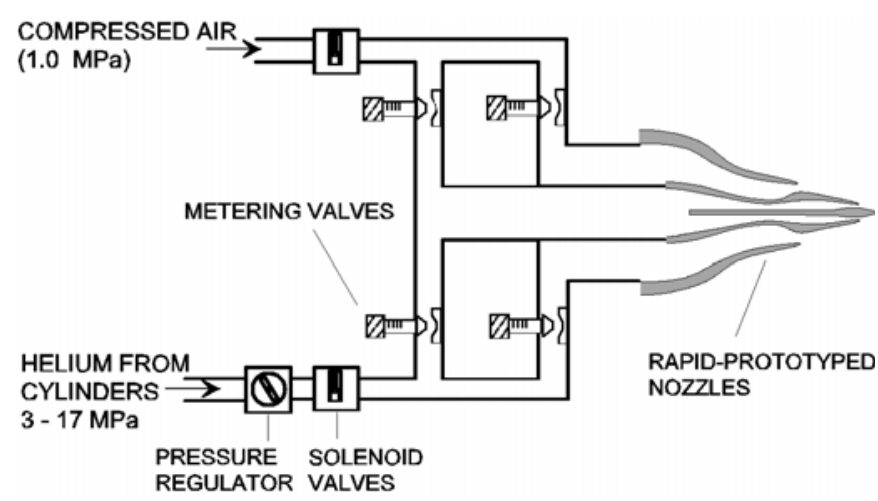

b)

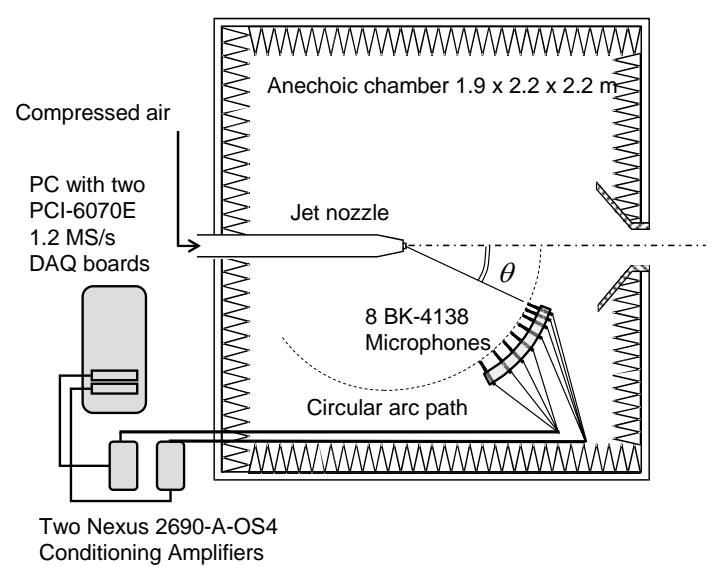

Fig. 2 UCI Jet Aeroacoustics lab. a) Flow facility; b) aeroacoustic measurement. 


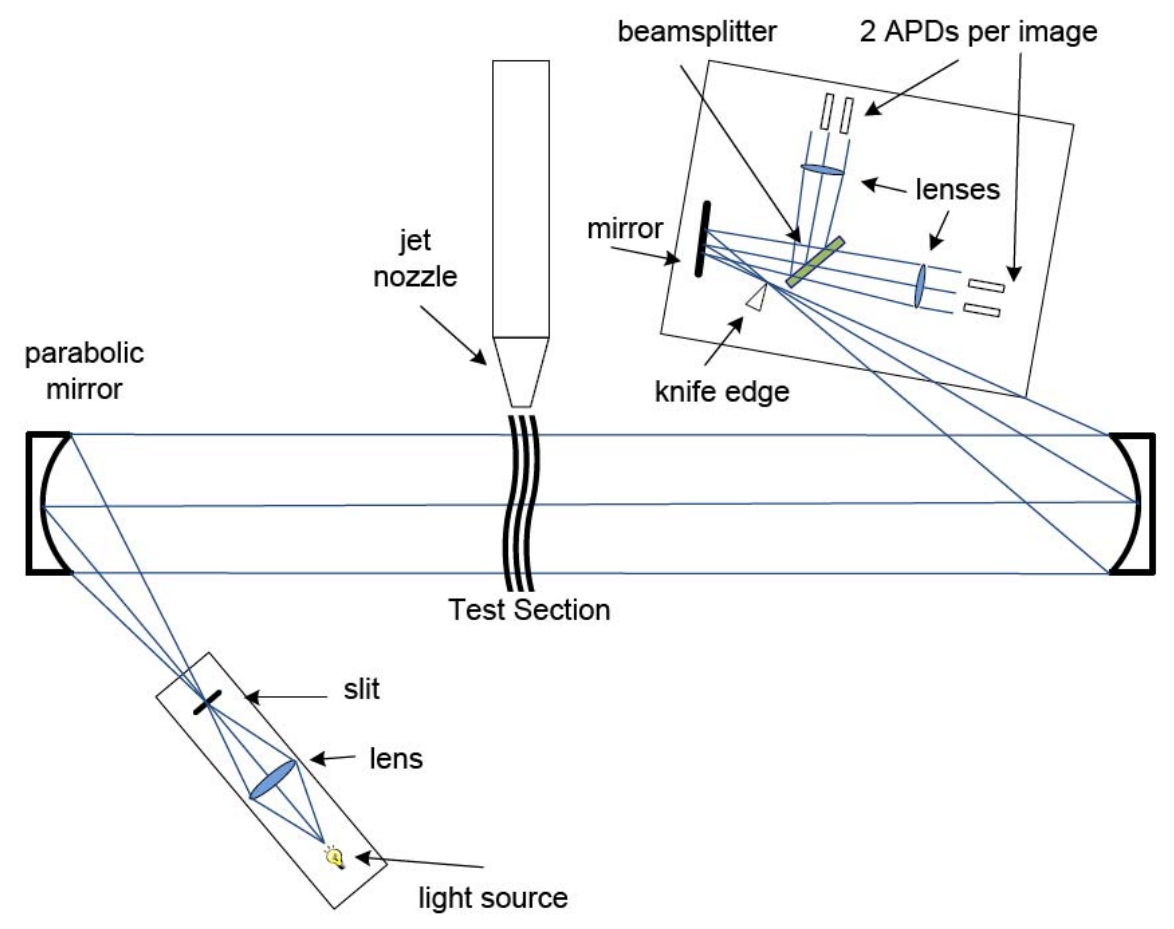

Fig. 3 Basic design of optical deflectometer.

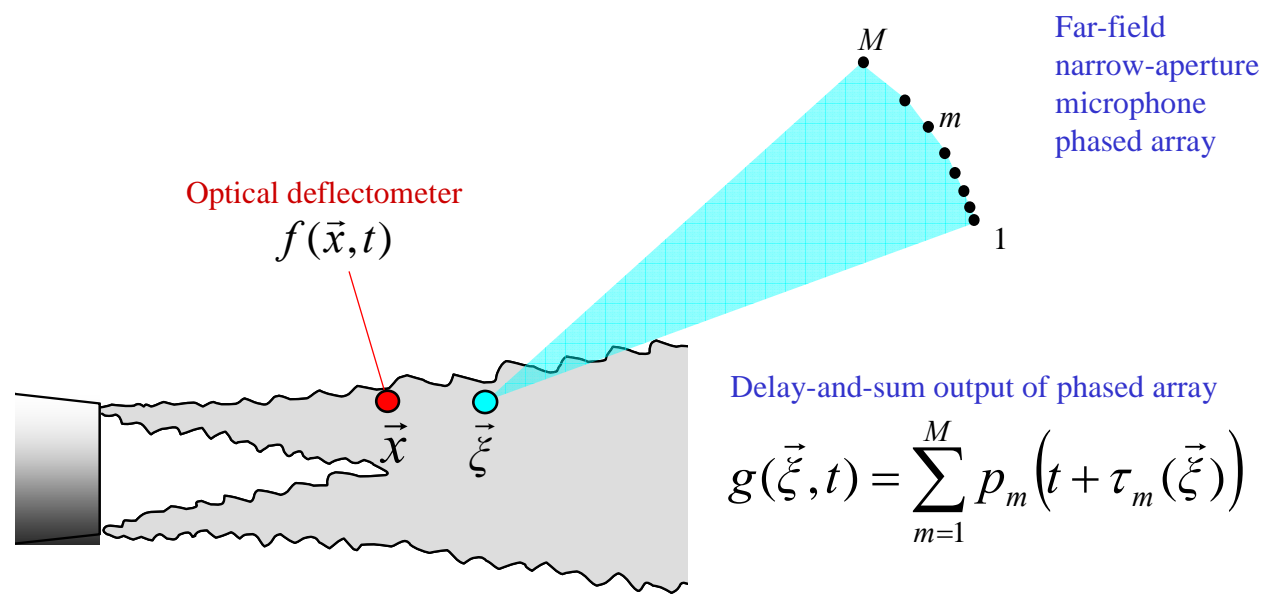

Fig. 4 Illustration of the correlation of the optical deflectometer (OD) signal with the delay-and-sum (DAS) output of the microphone array 


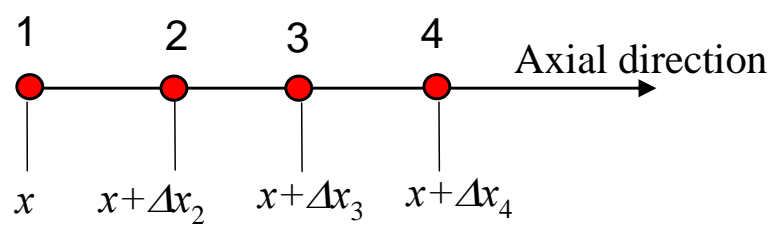

Fig. 5 Sequence of optical deflectometer (OD) probes used in OD beamforming.

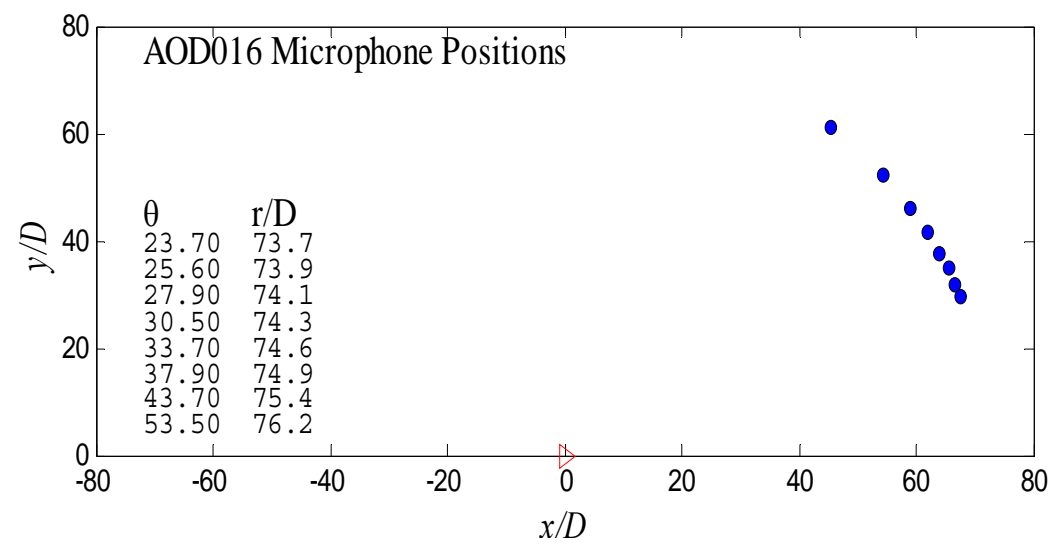

Fig. 6 Microphone positions.

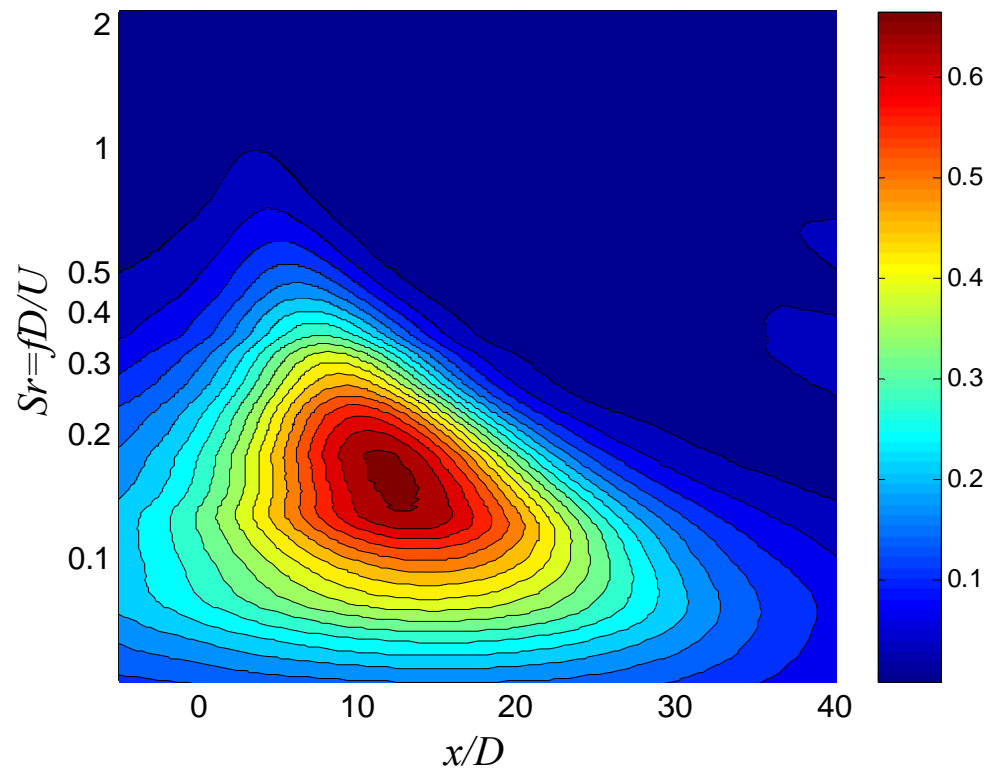

Fig. 7 Delay-and-sum beamform map of $M=1.75$ jet with microphones as shown in Fig.6. 


$$
\begin{aligned}
& y / D=0.48 \\
& (y-D / 2) / x=0
\end{aligned}
$$

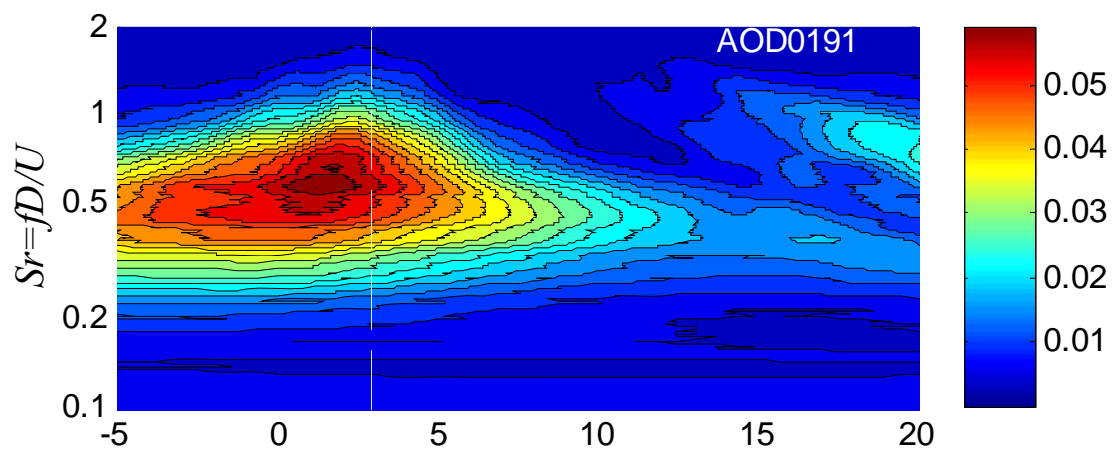

$y / D=0.68$

$(y-D / 2) / x=0.06$

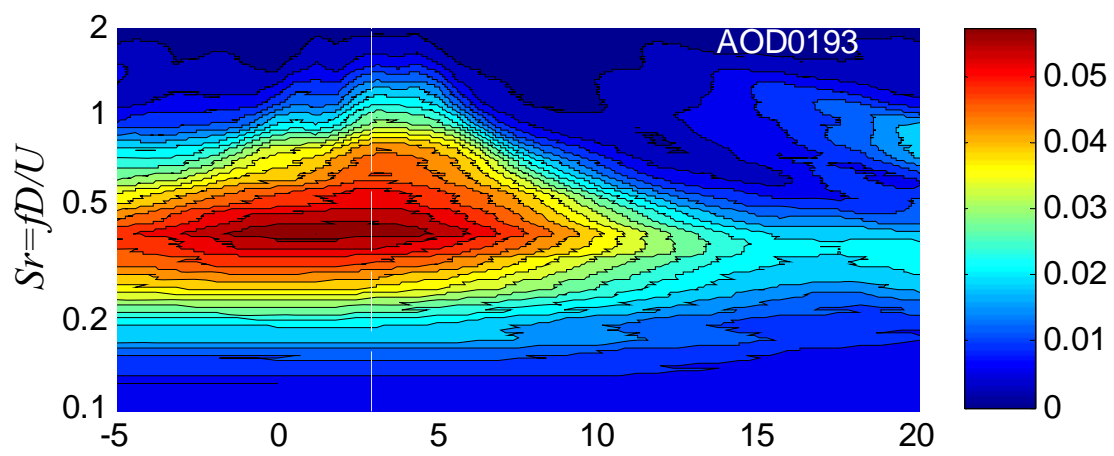

$y / D=1.18$

$(y-D / 2) / x=0.23$
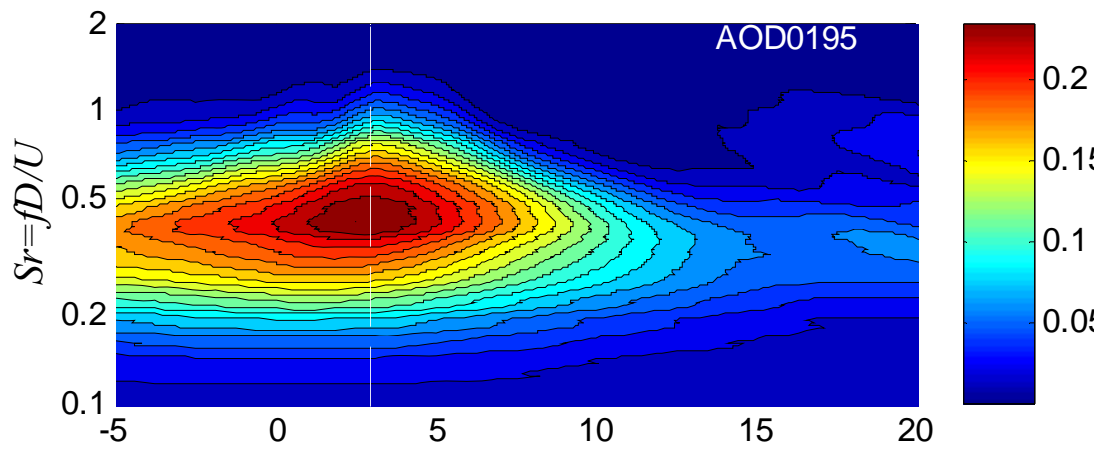

0.2

0.15

0.1

$y / D=1.98$

$(y-D / 2) / x=0.49$

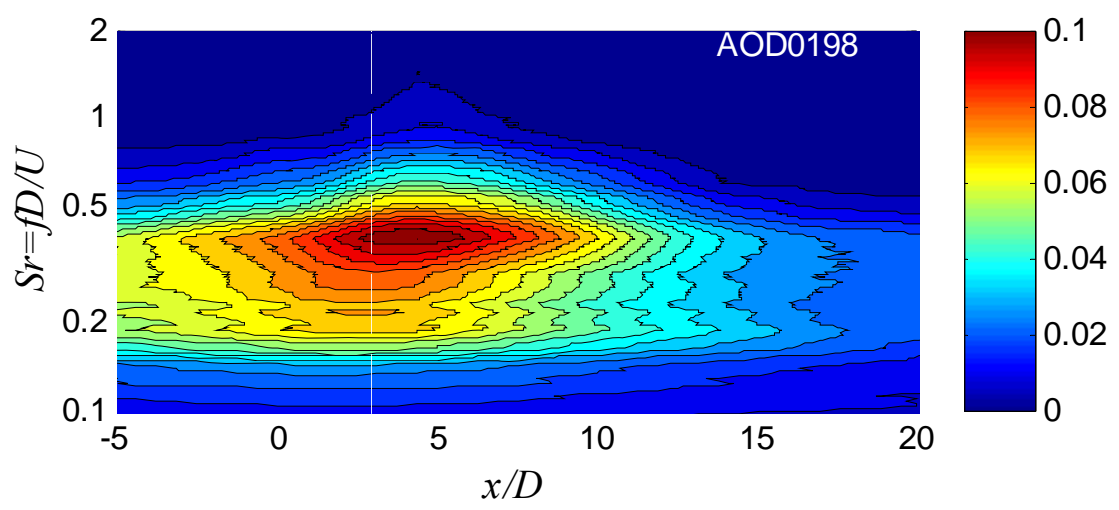

Fig. 8 Isocontours of coherence between single $O D$ probe at $x / D=3.0$ and delay-and-sum output of microphone array $\left(\mathrm{DAS}_{\text {mic }}\right)$. Dashed white line in this and following coherence plots shows the axial location of the probe. 


$$
\begin{aligned}
& y / D=0.48 \\
& (y-D / 2) / x=0
\end{aligned}
$$
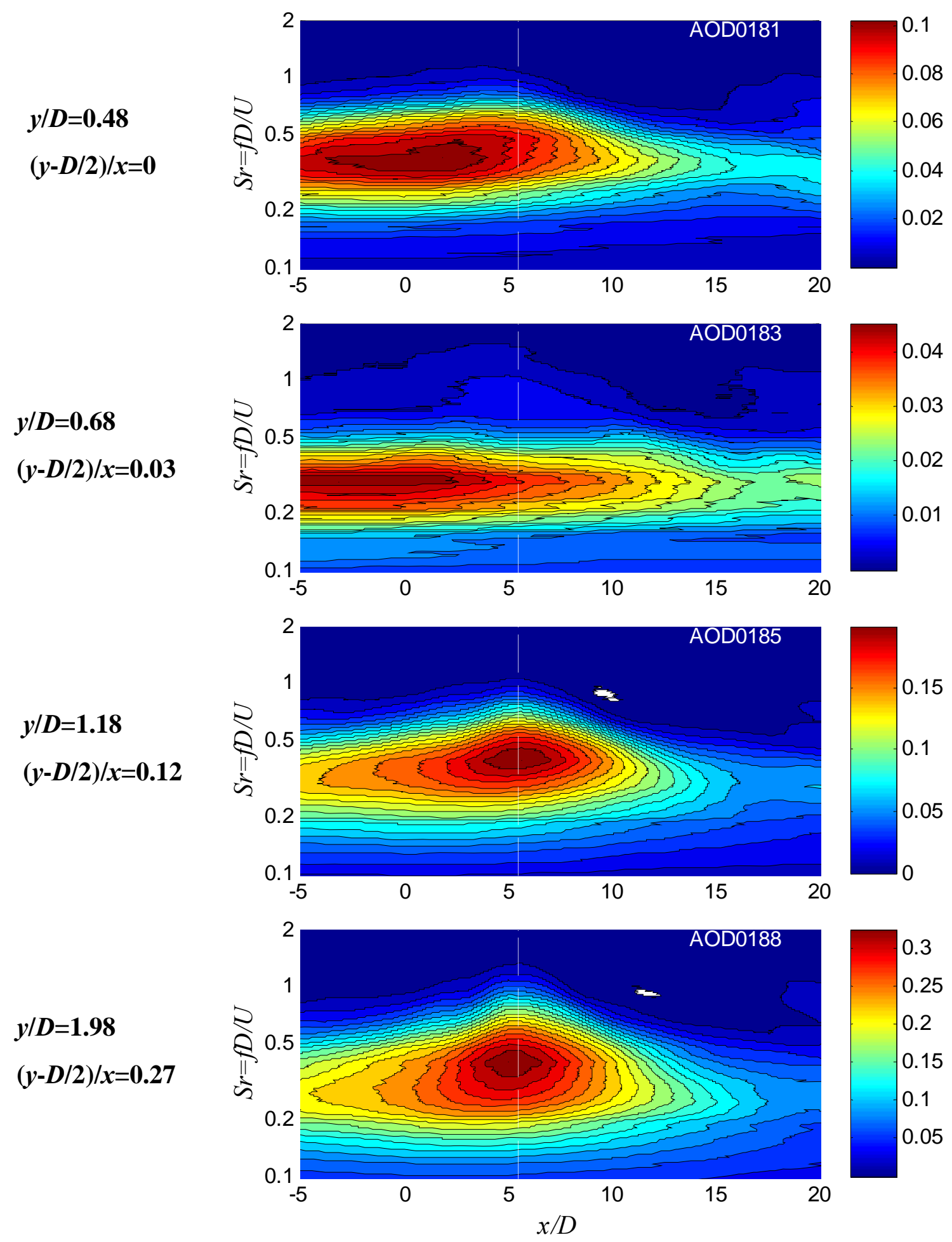

Fig. 9 Isocontours of coherence between single $O D$ probe at $x / D=5.5$ and delay-and-sum output of microphone array (DAS mic $_{\text {) }}$. 

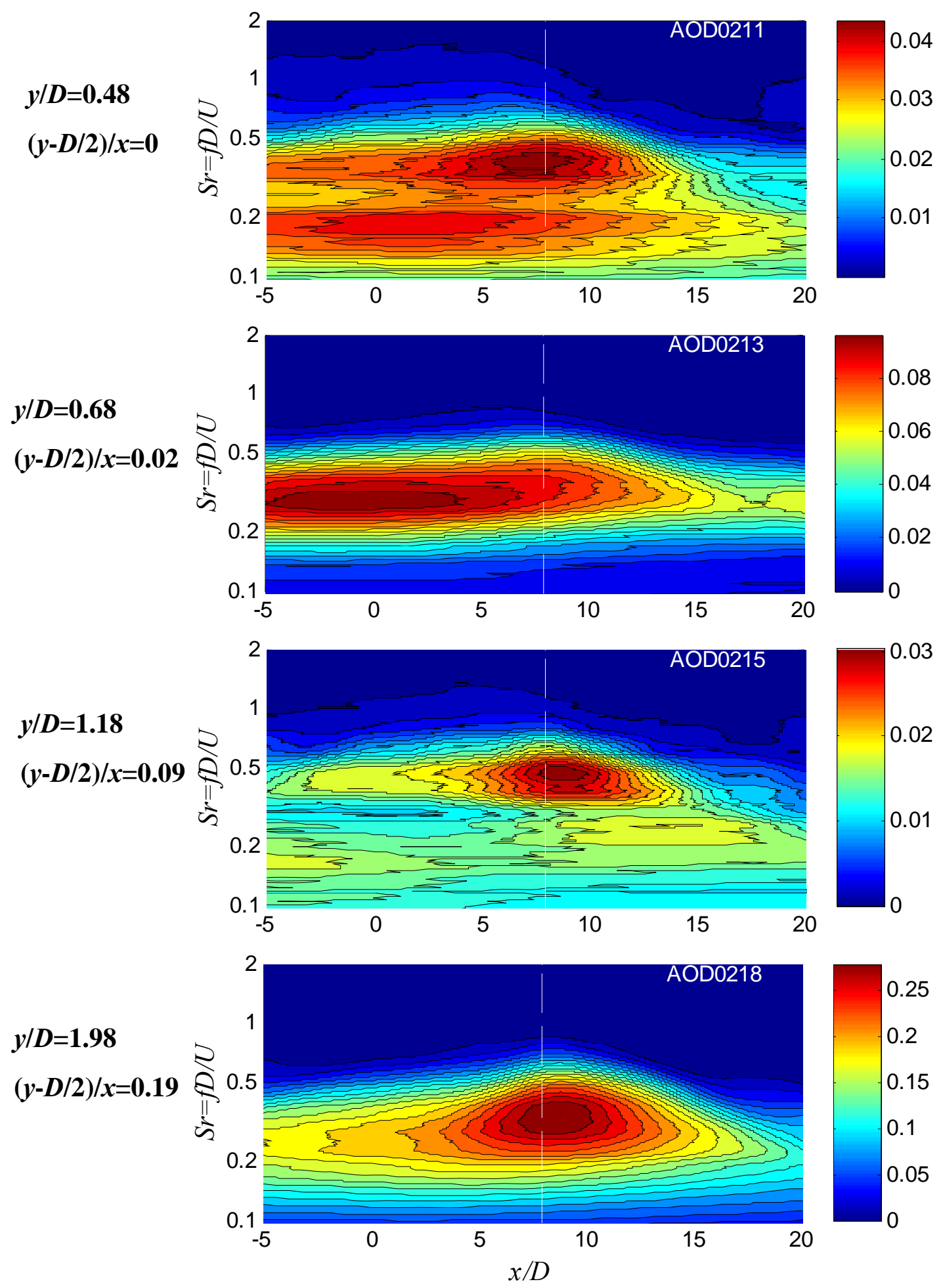

Fig. 10 Isocontours of coherence between single $O D$ probe at $x / D=8.0$ and delay-and-sum output of microphone array $\left(\right.$ DAS $\left._{\text {mic }}\right)$. 

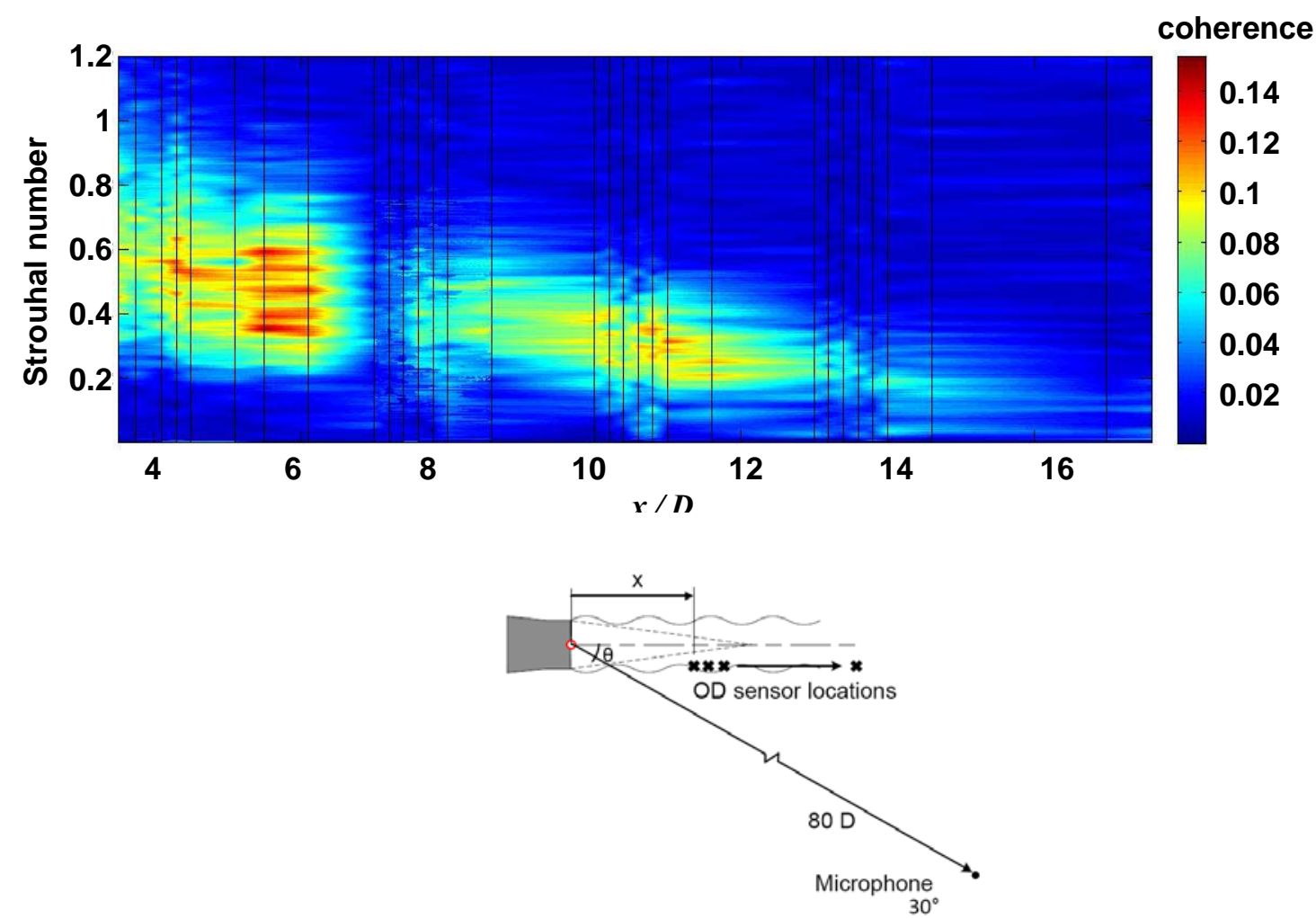

Figure 11: Isocontour plots of the coherence between an OD sensor scanning along the lipline of the jet and a microphone fixed in the far-field at $\theta=30^{\circ}$.

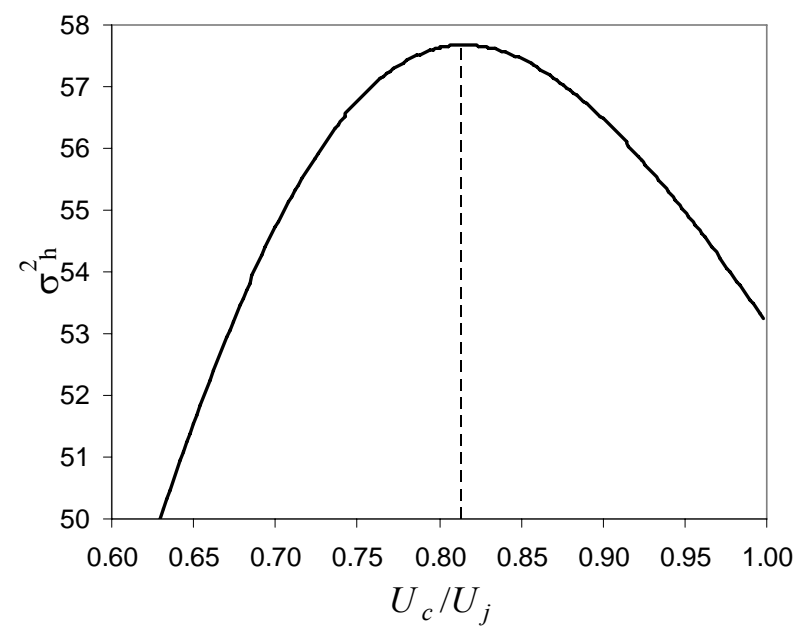

Fig. 12 Determination of convective velocity $U_{c}$ by maximization of the variance $\sigma_{h}^{2}$ of the delay-and-sum output of the optical deflectometer. 

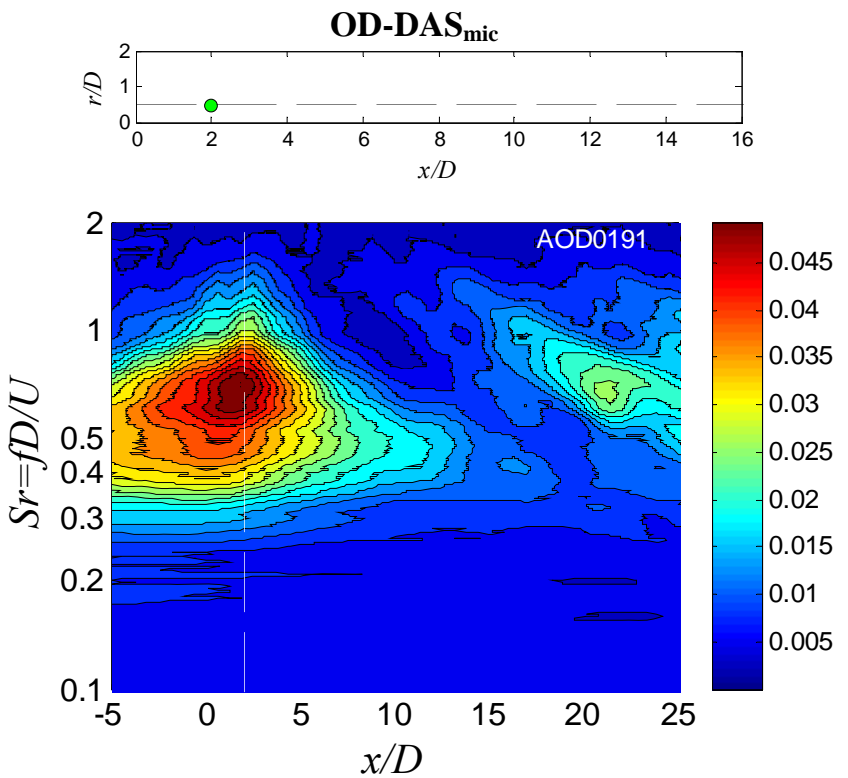
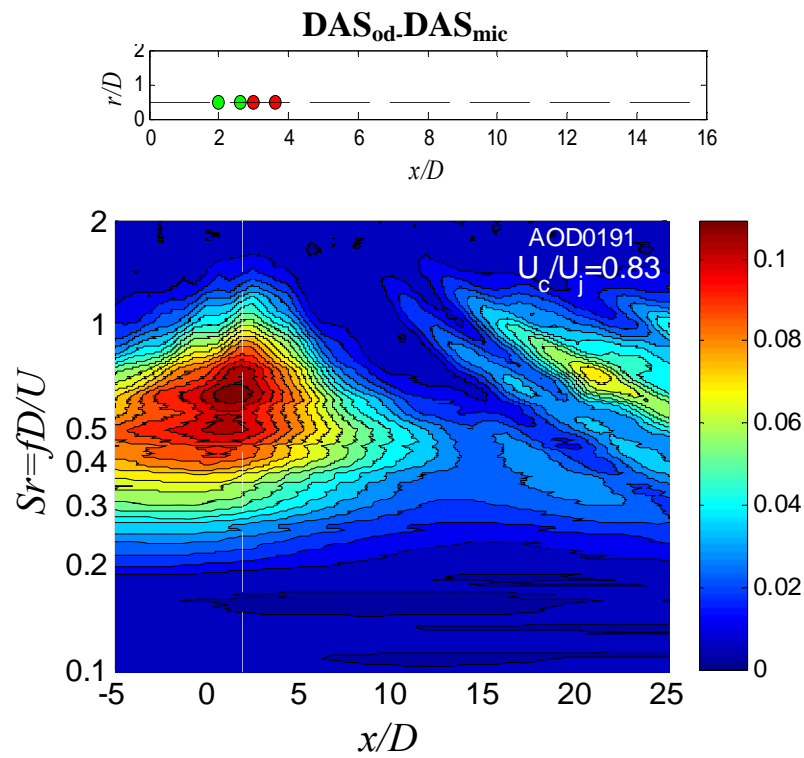

Fig. 13 Isocontours of coherence for OD-DAS ${ }_{\text {mic }}$ and $D A S_{\text {od }}-D A S_{\text {mic }}$. Reference probe at $x / D=2.0, r / D=0.48(l i p$ line). Computed convective velocity $U_{c}=0.83 U_{j}$ was used in $\mathrm{DAS}_{\text {od }}$ beamforming.
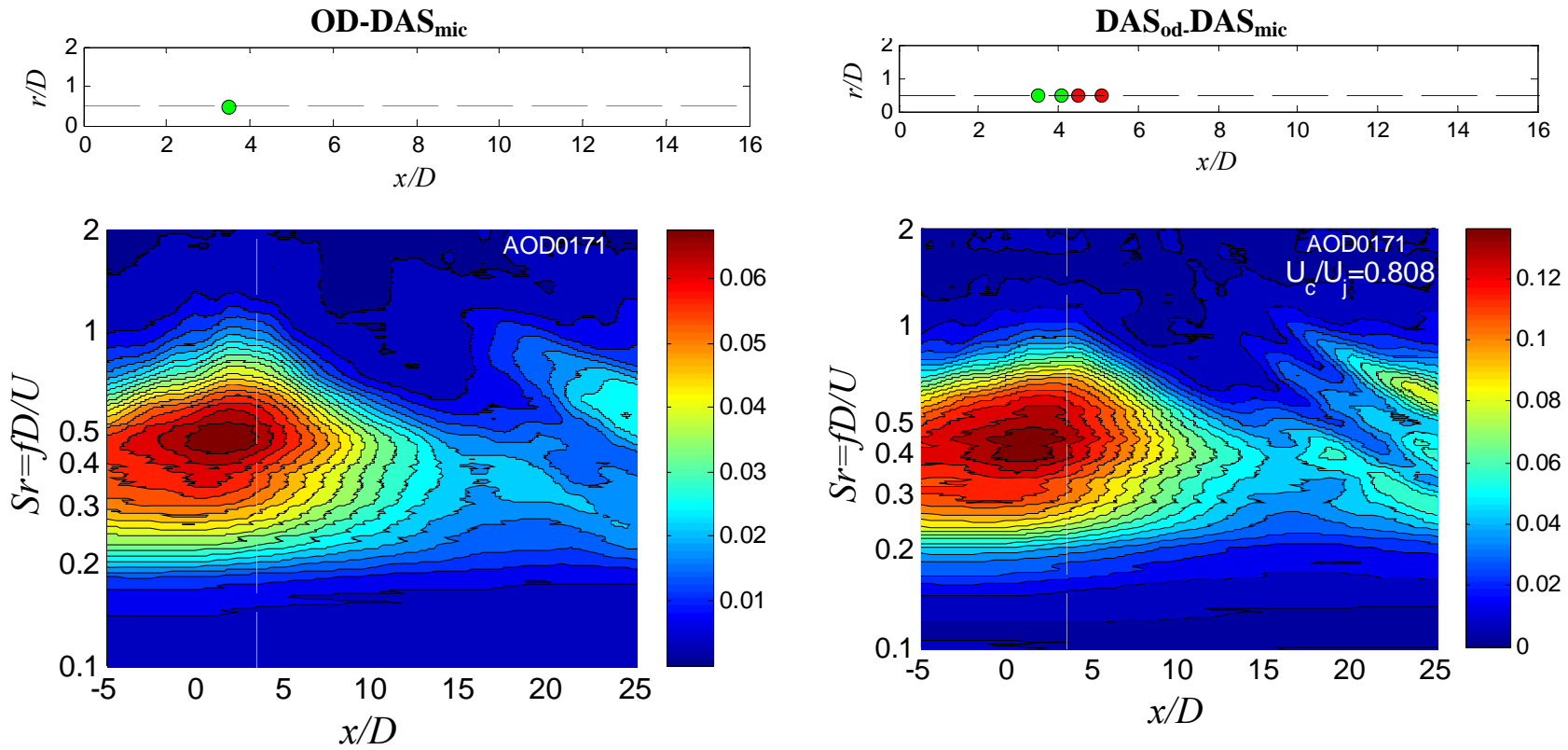

Fig. 14 Isocontours of coherence for OD-DAS ${ }_{\text {mic }}$ and DAS od $-D A S_{\text {mic }}$. Reference probe at $x / D=3.5, r / D=0.48($ lip line). Computed convective velocity $U_{c}=0.81 U_{j}$ was used in DAS od beamforming. 

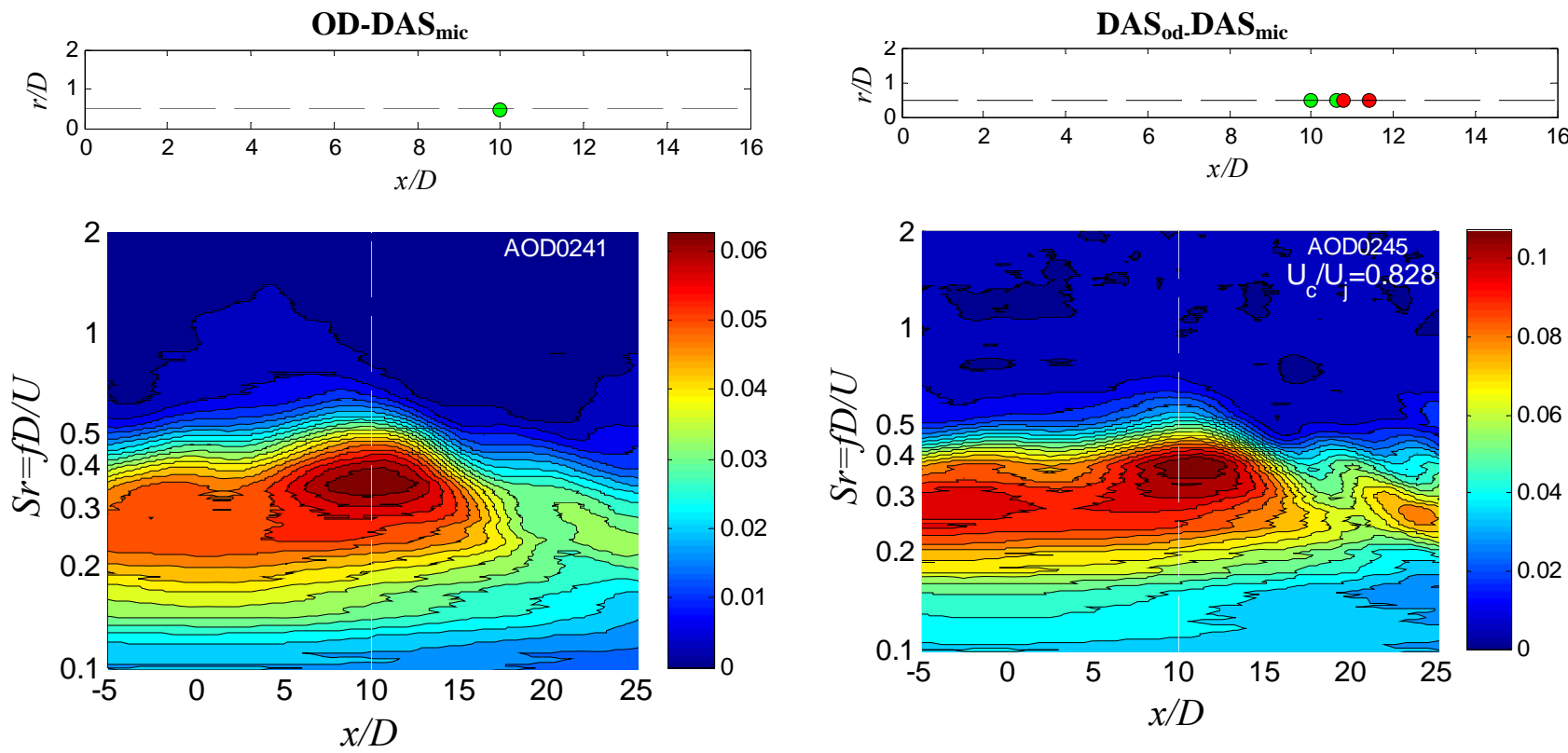

Fig. 15 Isocontours of coherence for OD-DAS ${ }_{\text {mic }}$ and DAS ${ }_{\text {od }}-D A S_{\text {mic }}$. Reference probe at $x / D=10, r / D=0.48($ lip line). Computed convective velocity $U_{c}=0.83 U_{j}$ was used in $\mathrm{DAS}_{\mathrm{od}}$ beamforming.
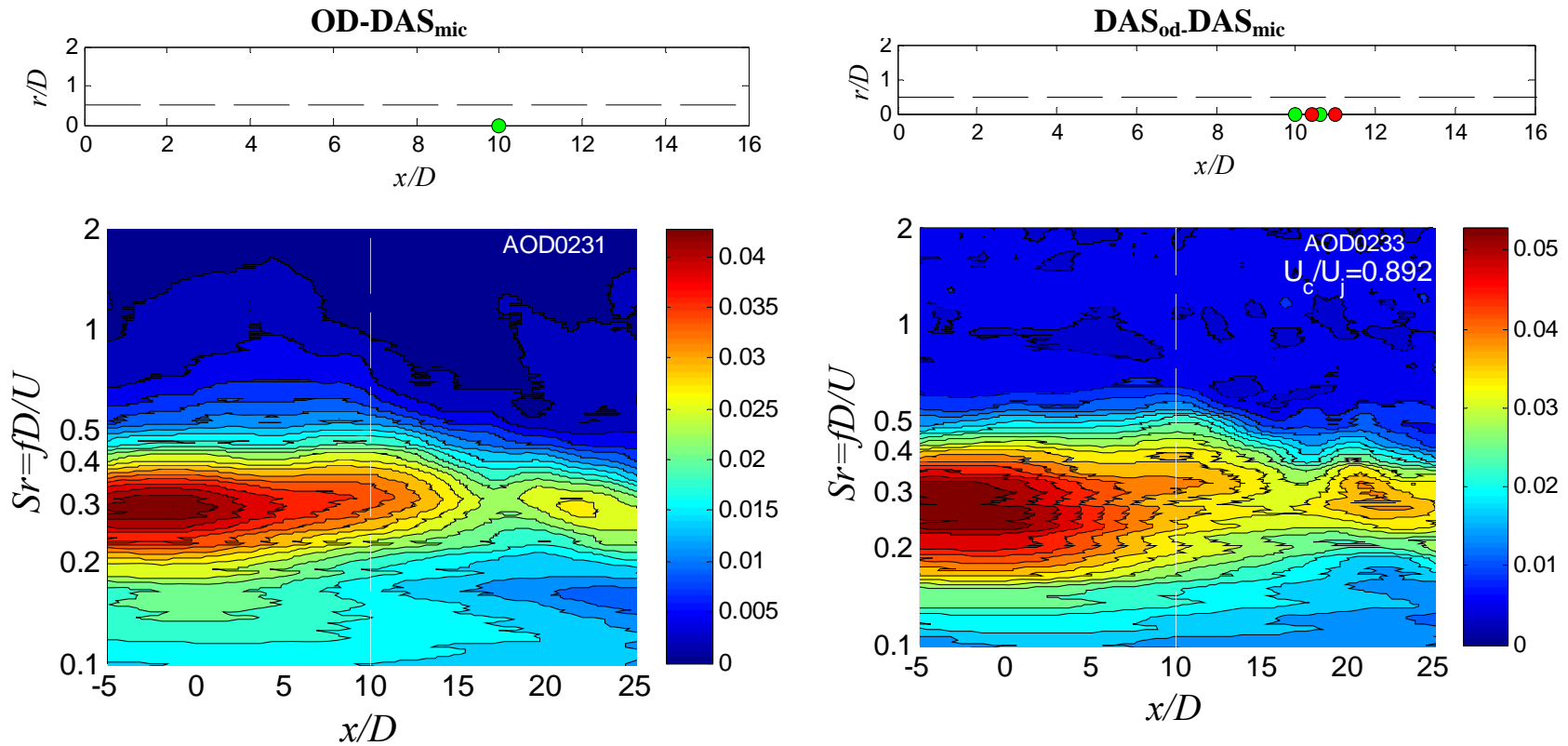

Fig. 16 Isocontours of coherence for $O D-D A S_{\text {mic }}$ and $D A S_{\text {od }}-D A S_{\text {mic }}$. Reference probe at $x / D=10, r / D=0$ (centerline). Computed convective velocity $U_{c}=0.89 U_{j}$ was used in DAS od beamforming. 


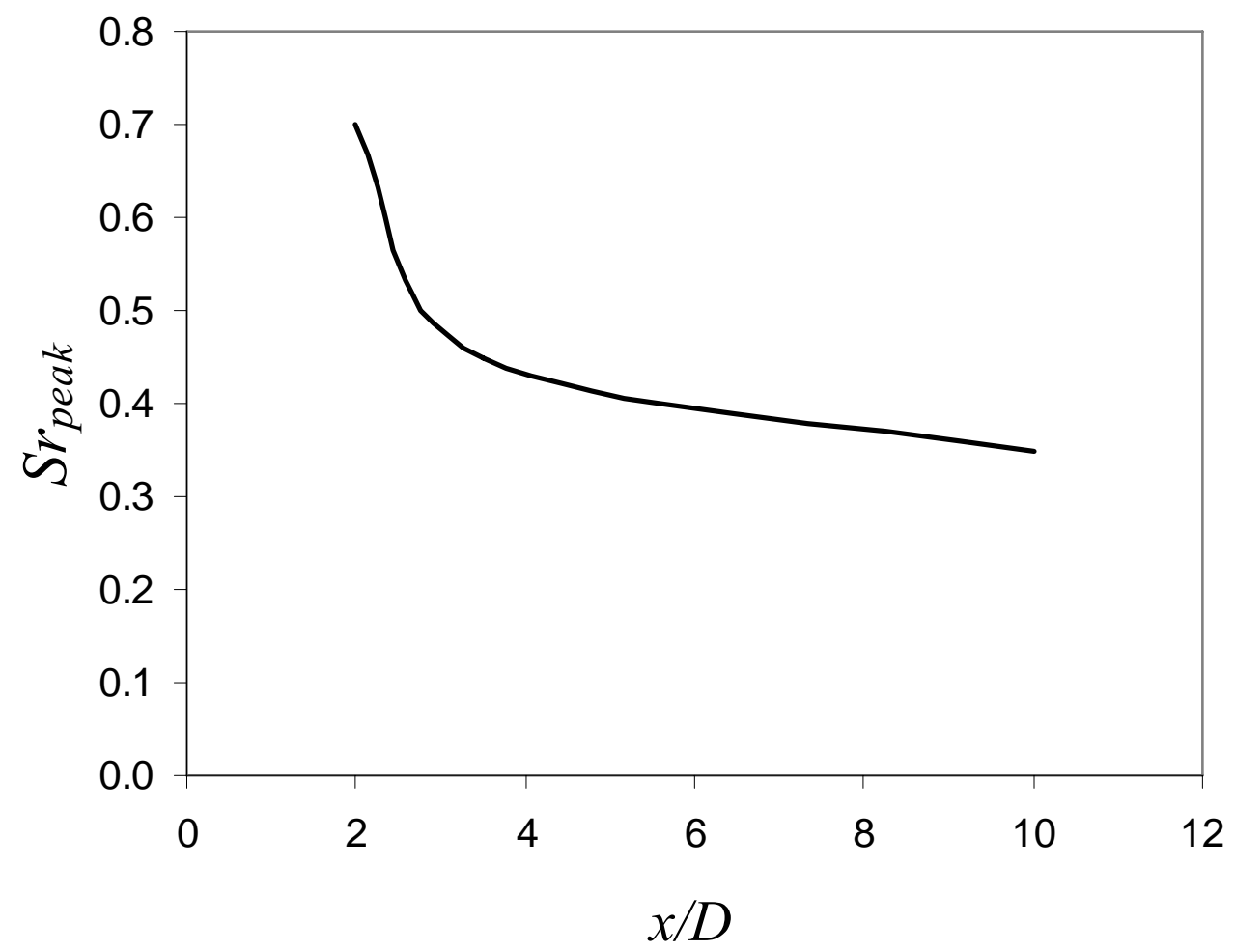

Fig. 17 Peak Strouhal number on jet lip line versus $x / D$. 